\title{
COVID-19: Dados Atualizados e sua Relação Com o Sistema Cardiovascular
}

\section{COVID-19: Updated Data and its Relation to the Cardiovascular System}

Filipe Ferrari ${ }^{1 \oplus}$

Programa de Pós-Graduação em Cardiologia e Ciências Cardiovasculares, Universidade Federal do Rio Grande do Sul, Hospital de Clínicas de Porto Alegre, ${ }^{1}$ Porto Alegre, $R S-$ Brasil

\section{Resumo}

Em dezembro de 2019, um novo coronavírus humano, chamado síndrome respiratória aguda grave do coronavírus 2 (SARS-CoV-2) ou nomeado doença de coronavírus (COVID-19) pela Organização Mundial da Saúde, surgiu na cidade de Wuhan, China. Difundido globalmente, é atualmente considerado pandêmico, com aproximadamente 3 milhões de casos no mundo no final de abril. Seus sintomas incluem febre, tosse, dor de cabeça e falta de ar, esse último considerado o sintoma principal. Por sua vez, acredita-se que haja uma relação entre o COVID-19 e danos ao músculo cardíaco, e pacientes com hipertensão e diabetes, por exemplo, parecem apresentar prognóstico pior. Portanto, o COVID-19 pode piorar em indivíduos com condições adversas subjacentes. Um número não negligenciável de pacientes internados com este vírus tinham doenças cardiovasculares ou cerebrovasculares. A resposta inflamatória sistêmica e distúrbios do sistema imunológico durante a progressão da doença podem estar por trás dessa associação. Além disso, o vírus usa os receptores da enzima conversora da angiotensina (ECA), mais especificamente da ECA2, para penetrar nas células; portanto, o uso de fármacos inibidores de ECA e bloqueadores de receptores de angiotensina pode causar um aumento nestes receptores, assim facilitando a entrada do vírus na célula. No entanto, não há evidências científicas que apóiem a interrupção desses medicamentos. Considerando que são fundamentais para o manejo de certas doenças crônicas, os riscos e benefícios da sua retirada devem ser cuidadosamente ponderados neste cenário. Finalmente, cardiologistas e profissionais de saúde devem estar cientes dos riscos de infecção e se proteger o máximo possível, dormindo adequadamente e evitando longos turnos de trabalho.

\section{Introdução}

Em dezembro 2019, na cidade de Wuhan, China, houve uma explosão de casos de pneumonia causados por um novo coronavírus, chamado síndrome respiratória aguda grave do coronavírus 2 (SARS-CoV-2), ${ }^{1}$ identificado como

\section{Palavras-chave}

Coronavirus; COVID-19; Síndrome Respiratória Aguda; Doenças Cardiovasculares/complicações; Miocardite; Doenças Infecciosas; Fatores de Risco/prevenção e controle.

Correspondência: Filipe Ferrari •

Universidade Federal do Rio Grande do Sul - Rua Mariana Prezzi, 617, 43B. CEP 95034460, Caxias do Sul, RS - Brasil

E-mail: ferrari.filipe88@gmail.com

Artigo recebido em 19/03/2020, revisado em 03/04/2020, aceito em 03/04/2020 o agente que causa a doença de coronavírus (COVID-19), nome oficialmente adotado pela Organização Mundial da Saúde. O COVID-19 é uma condição que pode afetar os pulmões, o trato respiratório e outros sistemas. Dados filogenéticos sugerem uma origem zoonótica, ${ }^{2}$ e tem sido demonstrado que a transmissão do vírus se dá de pessoa para pessoa. Tem sido detectado em escarro, saliva, e zaragatoas da garganta e nasofaríngeas. ${ }^{3}$ Portanto, pode espalhar-se por meio de pequenas gotículas liberadas pelo nariz e pela boca de indivíduos infectados. Os sintomas mais observados incluem febre, fadiga, tosse seca, congestão das vias aéreas superiores, produção de escarro, mialgia/artralgia com linfopenia e tempo prolongado de protrombina. ${ }^{4}$ No entanto, um dos sintomas principais é falta de ar.

Embora ainda sejam pouco conhecidas as evidências sobre os efeitos específicos do COVID-19 no sistema cardiovascular, há relatos de arritmias, lesão cardíaca aguda, taquicardia e uma alta carga de doença cardiovascular concomitantes nos indivíduos infectados, particularmente naqueles com mais comorbidades e fatores de risco que necessitam cuidados mais intensivos. ${ }^{5}$

O diagnóstico de SARS-CoV-2 pode ser feito pela morfologia microscópica, mas o método atualmente considerado o padrão-ouro é a detecção de ácido nucléico em zaragatoa nasal, amostras da garganta ou outras amostras do trato respiratório por reação em cadeia da polimerase (RCP) em tempo real, que é subsequentemente confirmado pelo sequenciamento de próxima geração. ${ }^{6}$

Finalmente, é necessário observar que o melhor tratamento continua sendo a prevenção, e medidas simples, tais como lavar as mãos com sabão, utilizar álcool em gel e desinfetar superfícies como celulares, desempenham um papel essencial na redução da propagação do vírus.

\section{Epidemiologia}

\section{Adultos e Idosos}

Dados mais recentes indicam que até o dia 23 de abril o número de casos confirmados de COVID-19 excediam 2.700.000 no mundo. ${ }^{7}$ Em 30 de janeiro de 2020, 9.976 casos de COVID-19 haviam sido relatados em pelo menos 21 países. ${ }^{8}$ Um mês depois, foram confirmados 83.652 casos, com 2.791 óbitos (mortalidade de 3,4\%). ${ }^{9}$ Foram relatados casos em 24 países, em 5 continentes. ${ }^{10}$ No Brasil, especificamente, até 3 de março, haviam sido registrados 488 casos suspeitos, em 23 estados. ${ }^{11}$ Adicionalmente, até 23 de abril haviam sido confirmados aproximadamente 49.500 casos e 3.313 óbitos por COVID-19 no Brasil. ${ }^{12} \mathrm{Na}$ Itália, em 20 fevereiro, um homem

DOI: https://doi.org/10.36660/abc.20200215 
jovem na região de Lombardia foi internado com pneumonia atípica que mais tarde provou ser COVID-19. Durante as 24 horas seguintes, houve mais 36 casos, nenhum dos quais havia tido contato com o primeiro paciente ou com outros casos confirmados de COVID-19.13 Infelizmente, apesar de medidas agressivas de contenção, a doença continua a se espalhar e o número de pacientes afetados está aumentando. A taxa de letalidade não está baixa, e predomina em pacientes idosos. ${ }^{12}$ Portanto, atenção especial deve ser dada a essa população.

Até 23 de abril o mundo já havia registrado 2.707.356 casos de COVID-19, incluindo 83.880 casos na China. Dos 190.743 óbitos da doença até esta data, 4.636 ocorreram na China. Já a Europa tinha registrado 1.193.276 casos, com 114.259 óbitos, ${ }^{7}$ tornando-se a região com o maior aumento de novas infecções a cada 24 horas. Várias regiões também registraram seus primeiros casos, incluindo Somália, Benin, Libéria e Bahamas. ${ }^{14}$

Existem incertezas sobre as estimativas do número real de pessoas infectadas, que seria crucial para determinar a severidade da infecção e a incidência de casos leves ou assintomáticos, bem como a sua possível transmissão. ${ }^{15}$

\section{Crianças}

Fatores epidemiológicos do COVID-19 entre crianças são escassos. Por meio de análise retrospectiva de crianças com uma idade média de 7 anos incluídas no Centro Chinês para Controle e Prevenção de Doenças entre 16 janeiro e 8 fevereiro 2020, Dong et al. ${ }^{16}$ observaram que houve 731 casos confirmados no laboratório e 1.412 casos suspeitos. Significativamente, mais de $90 \%$ destas crianças eram assintomáticas ou apresentaram apenas sintomas leves ou moderados. Esses dados chamam atenção para o fato de que não somente adultos e idosos mas também crianças de qualquer idade são suscetíveis ao COVID-19. Por este motivo, atenção e cuidados devem ser direcionados para a população inteira, sem distinção.

\section{COVID-19 e o Sistema Cardiovascular}

Infecções respiratórias e influenza podem desempenhar um papel importante no aumento a curto prazo do risco de infarto do miocárdio e acidente vascular cerebral isquêmico. ${ }^{17} \mathrm{SARS}-\mathrm{CoV}-2$ possui uma patogenicidade que pode aumentar danos no miocárdio causado por esta infecção viral. Os dados sugerem que lesão cardíaca aguda, choque e arritmia estavam presentes em 7,2\%, 8,7\% e $16,7 \%$ dos pacientes, respectivamente, e a sua prevalência era mais alta entre pacientes que necessitaram cuidados intensivos. ${ }^{10}$ Baseado no fato de que o vírus pode causar danos ao sistema cardiovascular, atenção cuidadosa deve ser dada à proteção cardiovascular durante o tratamento para COVID-19. ${ }^{18}$ De fato, doenças cardiovasculares e hipertensão foram associadas a uma taxa de letalidade aumentada de COVID-19 na China. ${ }^{19}$

Lesão do miocárdio associada a SARS-CoV-2 foi relatada em 5 dos primeiros 41 pacientes diagnosticados com COVID-19 em Wuhan, os quais apresentaram níveis de troponina cardíaca I de alta sensibilidade $>28 \mathrm{pg} / \mathrm{ml}^{20}$
Em outro estudo, realizado em 2019, Panhwar et al. ${ }^{21}$ observaram que infecção concomitante à influenza aumentou os riscos de pacientes internados com insuficiência cardíaca. Em uma pesquisa de 25 pacientes que haviam recuperado de infecção por SARS-CoV-1, quase a metade apresentou alterações no sistema cardiovascular, e 60\% apresentou distúrbios no metabolismo da glicose. ${ }^{22}$ Outro estudo incluiu 1.099 pacientes com COVID-19 confirmado, dos quais 173 apresentaram doença severa, com comorbidades de hipertensão $(23,7 \%)$, diabetes mellitus $(16,2 \%)$, doenças coronárias (5,8\%) e doença cerebrovascular (2,3\%). ${ }^{23}$

Em uma avaliação de dados de 138 pacientes internados por COVID-19 na China, o tempo mediano entre o primeiro sintoma e a dispneia foi de 5 dias, e de 7 dias entre o primeiro sintoma e a internação hospitalar. A tomografia computadorizada do tórax mostrou sombras fragmentadas bilaterais ou opacidade de vidro fosco nos pulmões de todos os pacientes. Aproximadamente $90 \%$ dos pacientes receberam terapia antiviral com oseltamivir, e mais de $60 \%$ receberam terapia antibacteriana com moxifloxacina. Trinta e seis paciente foram transferidos para a unidade de terapia intensiva devido a complicações, incluindo síndrome do desconforto respiratório agudo (61,1\%), arritmia (44,4\%) e choque (30,6\%). Os pacientes que necessitaram cuidados intensivos tinham idade mais avançada e maior probabilidade de apresentar comorbidades subjacentes, em adição à dispneia. Em 3 fevereiro, 34\% haviam recebido alta hospitalar e 6 morreram, representando uma mortalidade geral de $4,3 \% .^{10}$

Em pacientes com COVID-19, a incidência de sintomas cardiovasculares é alta, devido à resposta inflamatória sistêmica e distúrbios do sistema imunológico durante a progressão da doença. Por este motivo, pacientes com doenças cardiovasculares subjacentes que são infectados por COVID-19 podem apresentar prognóstico pior. Atenção especial deve, portanto, ser dada à proteção cardiovascular durante o tratamento de COVID-19.

\section{Receptores da Enzima conversora da Angiotensina e Bloqueadores de Receptores de Angiotensina}

O COVID-19 usa receptores da enzima conversora da angiotensina (ECA), mais especificamente a ECA2, para penetrar nas células. Tem sido hipotetizado que o uso de inibidores de ECA e bloqueadores de receptores de angiotensina (BRAs) possam aumentar esses receptores, assim facilitando a penetração do vírus. ${ }^{24}$

Em uma nota recente, a Sociedade Brasileira de Cardiologia enfatizou dados sobre a importância de tais fármacos, como inibidores de ECA e BRAs, uma vez que não há evidência clara apoiando a associação entre a terapia com esses medicamentos e prognóstico piorado da doença. ${ }^{25}$ Portanto, recomenda-se que os médicos cuidadosamente avaliem a relação risco-benefício antes de suspender os medicamentos, visto que são pilares fundamentais para o manejo de doenças crônicas, como hipertensão e insuficiência cardíaca. Da mesma forma, pacientes não devem interromper seu uso indiscriminadamente, sem antes consultar seus médicos. 


\section{Considerações finais}

O coronavírus é uma família de vírus que causam infecções respiratórias. O COVID-19 é uma doença grave que requer cuidados especiais. Indivíduos que apresentam febre, tosse e falta de ar devem procurar atendimento médico. Ao contrário do que muitos acreditam, o COVID-19 não é uma doença restrita aos idosos; jovens e crianças também podem ser infectados. Porém, pacientes idosos que têm doença cardiovascular infectados por COVID-19 podem apresentar prognóstico pior. Lavar as mãos frequentemente, usar álcool em gel, cobrir o nariz com a parte interna do braço e evitar ambientes aglomerados podem desempenhar um papel importante na redução da propagação do vírus e do agravamento da doença, especialmente em pacientes com doença cardiovascular.

\section{Contribuição dos autores}

Concepção e desenho da pesquisa, Obtenção de dados, Análise e interpretação dos dados, Redação do manuscrito

\section{Referências}

1. Zu ZY, Jiang MD, Xu PP, Chen W, Ni QQ, Lu GM, et al. Coronavirus disease 2019 (COVID-19): a perspective from China. Radiology. 2020 Feb 21:200490. [Epub ahead of print].

2. Ghinai I, McPherson TD, Hunter JC, Kirking HL, Christiansen D, Joshi K, et al. First known person-to-person transmission of severe acute respiratory syndrome coronavirus 2 (SARS-CoV-2) in the USA. Lancet. 2020;395(10230):1137-44

3. Phan T. Genetic diversity and evolution of SARS-CoV-2. Infect Genet Evol. 2020 Feb 21;81:104260.

4. Chan KW, Wong VT, Tang SCW. COVID-19: an update on the epidemiological, clinical, preventive and therapeutic evidence and guidelines of integrative Chinese-Western medicine for the management of 2019 novel coronavirus disease. Am J Chin Med. 2020 Mar 13:1-26. [Epub ahead of print].

5. American College of Cardiology. Coronavirus Disease 2019 (COVID-19) Provides Potent Reminder of the Risk of Infectious Agents [Internet], 2020 [citado 18 mar. 2020]. Disponível em: < https://www.acc.org/latest-incardiology/articles/2020/03/01/08/42/feature-coronavirus-disease-2019covid-19-provides-potent-reminder-of-the-risk-of-infectious-agents >

6. Guo YR, Cao QD, Hong ZS, Tan YY, Chen SD, Jin HJ, et al. The origin, transmission and clinical therapies on coronavirus disease 2019 (COVID-19) outbreak - an update on the status. Mil Med Res. 2020;7(1):11.

7. JOHNS HOPKINS. Coronavirus Resource Center. 2020. Disponível em: <https://coronavirus.jhu.edu/map.html>. Acessado em 23 de Abril, 2020.

8. Holshue ML, DeBolt C, Lindquist S, Lofy KH, Wiesman J, Bruce H, et al. First case of 2019 novel coronavirus in the United States. N Engl J Med. 2020;382(10):929-36.

9. Raoult D, Zumla A, Locatelli F, Ippolito G, Kroemer G. Coronavirus infections: epidemiological, clinical and immunological features and hypotheses. Cell Stress. 2020 Mar 2. [Epub ahead of print].

10. Wang D, Hu B, Hu C, Zhu F, Liu X, Zhang J, et al. Clinical characteristics of 138 hospitalized patients with 2019 novel coronavirus-infected pneumonia in Wuhan, China. JAMA. 2020 Feb 7. [Epub ahead of print]. e Revisão crítica do manuscrito quanto ao conteúdo intelectual importante: Ferrari F.

\section{Potencial conflito de interesses}

Declaro não haver conflito de interesses pertinentes.

\section{Fontes de financiamento}

O presente estudo não teve fontes de financiamento externas.

\section{Vinculação acadêmica}

Programa de Pós-Graduação em Cardiologia e Ciências Cardiovasculares, Universidade Federal do Rio Grande do Sul, Hospital de Clínicas de Porto Alegre.

\section{Aprovação ética e consentimento informado}

Este artigo não contém estudos com humanos ou animais realizados por nenhum dos autores.
11. Brasil.Ministério da Saúde. Plataforma Integrada de Vigilância em Saúde. Notificação de casos de doença pelo coronavírus 2019 (COVID-19); 2020. [citado 18 mar. 2020]. Disponível em: <http://plataforma.saude.gov.br/ novocoronavirus/>.

12. G1. Brasil tem 3.313 mortes e 49.492 casos de coronavírus, diz ministério. 2020. Disponível em: <https://g1.globo.com/bemestar/coronavirus/ noticia/2020/04/23/brasil-tem-3313-mortes-e-49492-casos-decoronavirus-diz-ministerio.ghtml>. Acessado em 23 de Abril, 2020.

13. Livingston E, Bucher K. Coronavirus disease 2019 (COVID-19) in Italy. JAMA. 2020 Mar 17. [Epub ahead of print].

14. Coronavirus latest: most infections and deaths are now outside China. Nature [Internet], 2020 [citado 18 mar. 2020]. Disponível em: <https://www.nature.com/articles/d41586-020-00154-w>.

15. Ng OT, Marimuthu K, Chia PY, Koh V, Chiew CJ, De Wang L, et al. SARSCoV-2 infection among travelers returning from Wuhan, China. N Engl J Med. 2020;382(15):1476-8.

16. Dong Y, Mo X, Hu Y, Qi X, Jiang F, Jiang Z, et al. Epidemiology of COVID-19 among children in China. Pediatrics. 2020;145(6):e20200702.

17. Elkind MS, Harrington RA, Benjamin IJ. Role of the American Heart Association in the Global COVID-19 Pandemic. Circulation. 2020 Mar 17. [Epub ahead of print].

18. Yang X, Yu Y, Xu J, Shu H, Xia J, Liu H, et al. Clinical course and outcomes of critically ill patients with SARS-CoV-2 pneumonia in Wuhan, China: a singlecentered, retrospective, observational study. Lancet Respir Med. 2020 Feb 24. [Epub ahead of print].

19. Wu Z, McGoogan JM. Characteristics of and important lessons from the Coronavirus Disease 2019 (COVID-19) outbreak in China: summary of a report of 72314 cases from the Chinese Center for Disease Control and Prevention. JAMA. 2020;323(13):1239-42.

20. Huang C, Wang Y, Li X, Ren L, Zhao J, Hu Y, et al. Clinical features of patients infected with 2019 novel coronavirus in Wuhan, China. Lancet. 2020;395(10223):497-506.

21. PanhwarMS, Kalra A, Gupta T, KolteD, KheraS, BhattDL, etal. Effect of influenza on outcomes in patients with heart failure. JACC Heart Fail. 2019;7(2):112-7. 
22. Wu Q, Zhou L, Sun X, Yan Z, Hu C, Wu J, et al. Altered lipid metabolism in recovered SARS patients twelve years after infection. Sci Rep. 2017;7(1):9110

23. Guan WJ, Ni ZY, HuY, Liang WH, Ou CQ, HeJX, et al. Clinical characteristics of coronavirus disease 2019 in China. N Engl J Med. 2020 Feb 28. [Epub ahead of print].
24. Zheng YY, Ma YT, Zhang JY, Xie X. COVID-19 and the cardiovascular system. Nat Rev Cardiol. 2020 Mar 5. [Epub ahead of print].

25. Sociedade Brasileira de Cardiologia. SBC Informa. Infecção pelo Coronavírus 2019 (COVID-19); 2020. [citado 18 mar. 2020]. Disponível em: <http://www.cardiol.br/sbcinforma/2020/20200313comunicado-coronavirus.html $>$.

Este é um artigo de acesso aberto distribuído sob os termos da licença de atribuição pelo Creative Commons 